\title{
The Role of Endosonography in the Staging of Gastrointestinal Cancers
}

\author{
Jin Woong Cho \\ Department of Gastroenterology, Presbyterian Medical Center, Jeonju, Korea
}

Endosonography (EUS) enables the acquisition of clear images of the gastrointestinal tract wall and the surrounding structures. EUS enables much greater accuracy for staging decisions compared to computed tomography. Surgery for esophageal cancer has a high rate of morbidity and mortality, and it is important to decide on an appropriate treatment method through pre-surgical evaluation. Minimal invasive surgery is widely used for the treatment of gastrointestinal cancer, and endoscopic submucosal dissection is a safe treatment method for early cancer of the gastrointestinal tract that does not result in lymph node metastasis. EUS is essential for pre-surgical evaluation for all esophageal cancers. The use of EUS can effectively reduce unnecessary surgeries and thereby allow for appropriate treatment planning for patients. A number of different diagnostic modalities are available, but EUS is still the mainstay for pre-surgical evaluation of esophageal cancer. The role of EUS for early stomach cancer treatment as a tool for determining the need for endoscopic resection and for pre-surgical assessment is increasing. Clin Endosc 2015;48:297-301

Key Words: Endosonography; Esophageal neoplasms; Stomach neoplasms; Rectal neoplasms

\section{INTRODUCTION}

Endosonography (EUS) enables the acquisition of clear images of the gastrointestinal tract wall and the surrounding structures. Because EUS shows in detail the origin and invasion level of tumors that grow in the intestinal wall, it enables much greater accuracy for staging decisions than computed tomography (CT).

The prognosis of esophageal cancer is poor because these tumors are usually detected in the advanced stage. Therefore, surgery is impossible in most cases, and those patients who do undergo surgery have a low chance of survival. Surgery for esophageal cancer has a high rate of morbidity and mortality, and it is important to decide on an appropriate treatment method through pre-surgical evaluation. Several diagnostic methods are available for pre-surgical evaluation of esopha-

Received: May 3, 2015 Accepted: June 23, 2015

Correspondence: Jin Woong Cho

Department of Gastroenterology, Presbyterian Medical Center, 365 Seowon-ro, Wansan-gu, Jeonju 560-750, Korea

Tel: +82-63-230-1321, Fax: +82-63-230-1309, E-mail: jeja-1004@hanmail.net

(cc) This is an Open Access article distributed under the terms of the Creative Commons Attribution Non-Commercial License (http://creativecommons.org/ licenses/by-nc/3.0) which permits unrestricted non-commercial use, distribution, and reproduction in any medium, provided the original work is properly cited. geal cancer, but EUS remains the most valuable tool.

Minimally invasive surgery is widely used for the treatment of gastrointestinal cancer, and endoscopic submucosal dissection (ESD) is a safe treatment method for early cancer of the gastrointestinal tract without lymph node metastasis. EUS is very useful for identifying patients with stomach cancer and esophageal cancer who will benefit from endoscopic treatment.

\section{ESOPHAGEAL CANCER}

\section{Characteristics of esophageal cancer and therapeu- tic approaches}

The prognosis of esophageal cancer is determined by the level of tumor invasion and lymph node metastasis. The 7th edition of the American Joint Committee on Cancer (AJCC) tumor node metastasis (TNM) cancer staging manual classifies esophageal squamous cell carcinoma and esophageal adenocarcinoma as differentiated and uses the number of metastasized lymph nodes rather than the location of the lymph nodes to determine the lymph node $\mathrm{N}$ stage.

In superficial esophageal cancer, invasion of the lymph nodes surrounding the cervical area and the celiac trunk is 
possible without invasion of the lymph nodes around the lesion itself. This significant characteristic of esophageal cancer makes it difficult to stage the tumors before surgery. Other challenges in treating esophageal cancer include a wide surgical area and high prevalence of disease and mortality due to surgery.

Lesions limited to the submucosa and muscularis propria can be resected with primary surgical excision. EUS is the most valuable and accurate examination for determining depth of invasion and lymph node metastasis in pre-surgical evaluation. It is used to ascertain those lesions that can be treated with endoscopy, and allows one to decide on a treatment by differentiating between patients who need surgical excision and those who need preoperative chemoradiation treatment. EUS-guided fine needle aspiration (EUS-FNA) is performed with excellent accuracy when lymph node metastases are suspected. ${ }^{2}$

\section{EUS imaging and clinical significance for evaluation of invasion depth}

The esophagus generally appears as five to seven layers on an EUS examination. Using a high ultrasound frequency of more than $20 \mathrm{MHz}$, EUS imaging of an esophagus can show nine layers. The prognosis of esophageal cancer without lymph node metastasis is based on the tumor size $\mathrm{T}$ stage, histologic findings, cell differentiation, and tumor location.

Irrespective of the presence of lymph node metastasis, cancer limited to the mucosa and submucosa is defined as superficial esophageal cancer. According to the TNM classification, superficial esophageal cancer is classified as T1s, T1a, or T1b. The mucosa is subdivided into M1, M2, and M3 ${ }^{3,4}$ Most M1 and M2 lesions do not have lymph node metastasis, and full recovery is possible through localized excision.

Most patients diagnosed with T2-T3 tumors on pre-surgical examination have lymph node metastasis in the surgical tissue. ${ }^{5}$ The prognosis of patients with T3 tumors is poor, and the rate of full recovery is less than $15 \%{ }^{6}$

\section{The role of EUS in T staging}

Esophageal cancer is evaluated preoperatively using positron emission tomography (PET) and CT, which have a very low accuracy for evaluating the depth of tumor invasion. With EUS, it is possible to locate lesions and accurately determine the T stage. According to a report by Puli et al., ${ }^{7}$ the sensitivity and specificity of EUS examination for esophageal cancer was $81.6 \%$ and $99.4 \%$ for $\mathrm{T} 1,81.4 \%$ and $96.3 \%$ for $\mathrm{T} 2$, 91.4\% and $94.4 \%$ for $\mathrm{T} 3$, and $92.4 \%$ and $97.4 \%$ for $\mathrm{T} 4$ staging, respectively, and was more accurate for advanced cancer than for early cancer.

EUS is very effective at differentiating $\mathrm{T} 1$ and $\mathrm{T} 2$ from $\mathrm{T} 3$ and T4 lesions and is an essential examination for planning surgical treatment. In $44 \%$ of cases, a change in treatment method was reported when using EUS examination rather than only using other examinations such as $\mathrm{CT}^{8}$ According to the 7th AJCC TNM staging, T4 lesions are divided into T4a (resectable) and T4b (unresectable). Patients with T4 lesions diagnosed using EUS generally had a very short survival period of less than 1 year irrespective of surgery.

Thosani et al. ${ }^{10}$ published the results of a meta-analysis on the use of EUS in staging early esophageal cancer, and reported the sensitivity and specificity as $85 \%$ and $87 \%$, respectively, for Tla and $86 \%$ for both sensitivity and specificity for T1b staging. A report on the analysis of early esophageal cancer surgery found that there was no lymph node metastasis in esophageal cancer lesions less than $3 \mathrm{~cm}$ in size confined to the lamina propria. ${ }^{11}$ To evaluate the mucosal subdivisions on EUS, a high ultrasound frequency of more than $20 \mathrm{MHz}$ has been used, but with considerably different results between observers.

\section{The importance of EUS in N staging}

In esophageal cancer, there is high rate of lymph node metastasis at an early stage, and the number of lymph node metastases influences prognosis. In upper esophageal cancer with lymph node metastasis, pre-surgical chemoradiotherapy is the standard treatment. Moreover, identification of the $\mathrm{N}$ stage is more accurate with EUS than with CT, particularly with the introduction of EUS-FNA. ${ }^{12}$

Lymph nodes that are more than $10 \mathrm{~mm}$ in diameter, have a clear border separating them from the surrounding tissue, are circular in form, and are homogeneously hypoechoic with an $85 \%$ chance of malignancy. However, because only $25 \%$ of malignant lymph nodes have all four of these characteristics, detection of diseased nodes is often difficult. Vazquez-Sequeiros et al. ${ }^{13}$ added three characteristics-celiac axis lymph nodes, five or more lymph nodes found on EUS, and T3 or T4 tumors-to the previous four standards and diagnosed lymph node metastasis if three of the conditions were met, achieving $86 \%$ accuracy.

The AJCC classifies the N stage as N1 (1 to 2), N2 (3 to 6), and N3 ( $\geq 7$ ) according to the number of metastasized lymph nodes. On pre-surgical EUS, the sensitivity and specificity for $\mathrm{N}$ staging was $60 \%$ to $97 \%$ and $40 \%$ to $100 \%$, respectively. ${ }^{14}$ Generally, the possibility of malignant lymph nodes increases concurrently with higher $\mathrm{T}$ stage, and lymph nodes closer to the tumor have a greater possibility of being malignant. On evaluation of the $\mathrm{N}$ stage in pre-surgical esophageal cancer, the accuracy of CT and EUS on average was $51 \%$ and $74 \%$, respectively, making EUS significantly more accurate. ${ }^{12}$ The $\mathrm{N}$ staging accuracy increases when EUS-FNA is used, and in 
one report, the accuracy was $99.4 \% .{ }^{15}$ In a study by Chen and Eloubeidi ${ }^{16}$ there was a higher survival rate corresponding to fewer malignant lymph nodes discovered on EUS. When the number of malignant lymph nodes was divided into 0,1 to 2 , and more than 2 , the survival time was, respectively, $66,14.5$, and 6.5 months. ${ }^{16}$

Celiac lymph node metastasis is linked to poor prognosis. Most celiac lymph nodes found on EUS are metastasized nodules. Those patients with celiac lymph nodes less than $2 \mathrm{~cm}$ in size have a better prognosis than those with lymph nodes larger than $2 \mathrm{~cm}$. The sensitivity of EUS-FNA for celiac lymph node diagnosis was $72 \%$ to $83 \%$, the specificity was $85 \%$ to $98 \%$, and the diagnostic accuracy was $94 \%{ }^{17-20}$

\section{The limitations of EUS in esophageal cancer}

The use of EUS ensures a decrease in unnecessary surgeries and allows for the appropriate treatment for patients. However, despite these encouraging research results, there are a few limitations.

In esophageal cancer, the peripheral margin of the lesion is difficult to evaluate through diagnostic endoscopy alone, and the range of lesions observed on chromoendoscopy is generally wider than the area observed without Lugol solution staining. Evaluating the depth of invasion with EUS is performed by directly approaching the lesion observed on the endoscopy image with the ultrasound probe, and it is therefore difficult to accurately evaluate all areas of the lesion in wide lesions.

The EUS examination is limited in large lesions where the esophagus is obstructed due to a mass, and patients who undergo procedures to dilate the narrowed segment are likely to experience perforation. Owing to the risk of aspiration pneumonia in the cervical esophagus, EUS is limited to using water or jelly.

\section{GASTRIC CANCER}

\section{Use of EUS in the treatment of gastric cancer}

In the treatment of early gastric cancer, ESD is the safest and most effective treatment. As experience in ESD increases and various accessories develop, it is increasingly possible to remove lesions irrespective of the area and size. Lesions that can be removed remove via ESD are those that have not deeply invaded the submucosa and do not have lymph node metastasis. Many imaging methods have been used, but there are no diagnostic methods for predicting lymph node metastasis accurately in stomach cancer.

EUS is useful in choosing a treatment approach for lesions in which invasion to the submucosa is suspected upon examination of the gross endoscopic appearance or for gastric adenomas or mucosal cancers detected by pathologic examination. Moreover, in cancers of a size where ESD is likely to be an extensive process, EUS may be used before ESD. In the case of large lesions, EUS may find sites of blood vessels within a tumor before ESD, preventing excessive blood loss. However, when ulcers or fibrotic lesions are present, the depth of invasion is most likely exaggerated and some patients who might otherwise receive endoscopic resection (ER) may be treated by unnecessary surgery. The pattern analysis by Kida is useful for such patients.

\section{The role of EUS in T staging}

Classifying lesions with submucosal invasion before surgery ensures an accurate prognosis and is necessary for surgical planning. When restricted to the results of the endoscopy examination alone, the evaluation of invasion depth has many limitations. Puli et al..$^{21}$ published the results of a meta-analysis including 22 studies to evaluate the usefulness of EUS in stomach cancer, and the sensitivity and specificity by stage were, respectively, $88.1 \%$ and $100 \%$ for $\mathrm{T} 1,82.3 \%$ and $95.6 \%$ for T2, $89.7 \%$ and $94.7 \%$ for T3, and $99.2 \%$ and $96.7 \%$ for T4 staging. Incidentally, EUS for T stage detection was more accurate in advanced cancer than in early cancer. ${ }^{21}$ Mocellin et al. ${ }^{22}$ analyzed the distinction between T3-4 and T1-2 lesions, and EUS showed $86 \%$ sensitivity and $91 \%$ specificity.

To determine suitable patients for ER using EUS, it is necessary to accurately interpret EUS images. Mouri et al. ${ }^{23}$ evaluated the usefulness of EUS for determining the applicability of ESD and visualizing invasion depth in early gastric cancer. The EUS-mucosa (M) and EUS-M/submucosa (SM) borders had no tumor cells in the vertical margins in all patients who underwent ESD, and were good indicators for $\mathrm{ER}^{23}$

\section{The diagnostic value of EUS in $\mathrm{N}$ stage}

The AJCC 7th edition TNM classification of gastric cancer made an important change to the classification of lymph node metastasis lesions. The previous edition classified 1 to 6 lymph node lesions as $\mathrm{N} 1$, whereas the present edition defines $\mathrm{N} 1$ as having 1 to 2 lymph node lesions and $\mathrm{N} 2$ as 3 to 6 metastasized lymph nodes. The accuracy of EUS pre-surgical N stage evaluation is approximately $65 \%$ to $95 \%{ }^{24} \mathrm{Car}-$ doso et al. ${ }^{25}$ conducted a meta-analysis regarding pre-surgical $\mathrm{N}$ stage evaluation (N0 vs. $\mathrm{N}+$ ), and they reported an accuracy of $64 \%$, sensitivity of $74 \%$, and specificity of $80 \%$. The accuracy was unsatisfactory because of the difficulty in identifying changes in the lymph nodes due to inflammation and metastasized lymph nodes, and because there is a low possibility of detecting metastasized lymph nodes that are distant from the lesion. 


\section{EUS vs. CT in preoperative evaluation}

The mainstay examination before gastric cancer surgery is CT. Studies regarding early cancer for penetration depth using CT have reported unsatisfactory results, but the development of multidetector computed tomography (MDCT) has increased the accuracy of T stage evaluation. EUS is the preferred modality for determining invasion depth, but is limited in evaluating distant metastasis. The accuracy of $\mathrm{T}$ stage determination with EUS and CT was $65 \%$ to $92 \%$ and $77.1 \%$ to $88.8 \%$, respectively. ${ }^{26}$ For $\mathrm{N}$ stage evaluation, the sensitivity and specificity were, respectively, $71 \%$ and $49 \%$ for EUS, and $80 \%$ and $78 \%$ for MDCT. $^{27}$

Peritoneal invasion must be detected on pre-surgical examination to avoid unnecessary surgery. In diagnosing T4 tumors (serosa), the sensitivity and specificity of EUS were $77.8 \%$ to $100 \%$ and $67.9 \%$ to $100 \%$, respectively, and that of CT was $82.8 \%$ to $100 \%$ and $80 \%$ to $96.8 \%$, respectively. ${ }^{26}$ For detecting peritoneal metastases, the sensitivity and specificity were, respectively, $34 \%$ and $96 \%$ for EUS, $33 \%$ and $99 \%$ for CT, and $28 \%$ and $97 \%$ for PET. $^{28}$

\section{RECTAL CANCER}

The accuracy of EUS for pre-surgical invasion depth evaluation was $80 \%$ to $95 \%$. In identifying the $\mathrm{T}$ stage, the sensitivity was higher for advanced lesions than early cancer. An analysis of 42 studies revealed that the sensitivity and specificity were, respectively, $87.8 \%$ and $98.3 \%$ for $\mathrm{T} 1,80.5 \%$ and $95.6 \%$ for $\mathrm{T} 2$, $96.4 \%$ and $90.6 \%$ for $\mathrm{T} 3$, and $95.4 \%$ and $98.3 \%$ for $\mathrm{T} 4{ }^{29}$

In the treatment of rectal cancer, the pre-surgical diagnosis must have a high accuracy rate for T3 and T4 tumors that need neoadjuvant chemoradiation treatment. EUS, CT, and magnetic resonance imaging (MRI) have long been the mainstay of diagnosis for this purpose. A recent meta-analysis including 90 studies on T1 and T2 lesions found that while EUS and MRI have similar sensitivity, the specificity of EUS was higher (86\% vs. $69 \%$ ). In T3 tumors, EUS sensitivity was significantly higher than that of MRI and $\mathrm{CT}^{30}$

EUS cannot produce accurate results when there is a narrowing due to a mass or when the lesions are large and protruding. Furthermore, advancements in MRI technology have increased its accuracy for invasion depth evaluation compared with EUS, and so the role of EUS in rectal cancer evaluation has been decreasing. ER is an effective method for treating rectal tumors limited to the mucosa with a submucosal invasion depth of less than 1,000 $\mu \mathrm{m}$. EUS can be used as a diagnostic method for lesions that are difficult to classify using other endoscopic modalities.

EUS accuracy is low for nodal staging, as with other non-esophageal cancers. Because it cannot detect benign lymph nodes around the rectum, lymph node visualization is suggestive of metastasis. Recent studies have found that the role of EUS in nodal staging decisions is limited.

\section{CONCLUSIONS}

EUS is essential for pre-surgical evaluation for all esophageal cancers. The use of EUS reduces the number of unnecessary surgeries and allows for appropriate treatment. Different diagnostic modalities are available, but EUS is the mainstay for the pre-surgical evaluation of esophageal cancer.

The use of EUS as a tool for determining the need for ER in early stomach cancer treatment has increased. In rectal cancer cases, the role of EUS in identifying T3 or T4 lesions has reduced.

Conflicts of Interest

The author has no financial conflicts of interest.

\section{REFERENCES}

1. Edge SB; American Joint Committee on Cancer. AJCC Cancer Staging Manual. 7th ed. New York: Springer; 2010. 103p.

2. Kim HG, Moon JS, Cho HW. Esophagus and mediastinum. In: KSGE EUS committee, ed. Textbook of Endoscopic Ultrasonography. Seoul: JIN; 2011. p. 34-63.

3. Endo M, Yoshino K, Kawano T, Nagai K, Inoue H. Clinicopathologic analysis of lymph node metastasis in surgically resected superficial cancer of the thoracic esophagus. Dis Esophagus 2000;13:125-129.

4. Leers JM, DeMeester SR, Oezcelik A, et al. The prevalence of lymph node metastases in patients with $\mathrm{T} 1$ esophageal adenocarcinoma a retrospective review of esophagectomy specimens. Ann Surg 2011;253:271278.

5. Stiles BM, Mirza F, Coppolino A, et al. Clinical T2-T3N0M0 esophageal cancer: the risk of node positive disease. Ann Thorac Surg 2011;92:491496.

6. Rice TW, Zuccaro G Jr, Adelstein DJ, Rybicki LA, Blackstone EH, Goldblum JR. Esophageal carcinoma: depth of tumor invasion is predictive of regional lymph node status. Ann Thorac Surg 1998;65:787-792.

7. Puli SR, Reddy JB, Bechtold ML, Antillon D, Ibdah JA, Antillon MR. Staging accuracy of esophageal cancer by endoscopic ultrasound: a meta-analysis and systematic review. World J Gastroenterol 2008;14:14791490.

8. Gheorghe C, Stanescu C, Gheorghe L, et al. Preoperative noninvasive EUS evaluation in patients with esophageal cancer considered for esophagectomy. J Gastrointestin Liver Dis 2006;15:137-141.

9. Fockens P, Kisman K, Merkus MP, van Lanschot JJ, Obertop H, Tytgat GN. The prognosis of esophageal carcinoma staged irresectable (T4) by endosonography. J Am Coll Surg 1998;186:17-23.

10. Thosani N, Singh H, Kapadia A, et al. Diagnostic accuracy of EUS in differentiating mucosal versus submucosal invasion of superficial esophageal cancers: a systematic review and meta-analysis. Gastrointest Endosc 2012;75:242-253.

11. Choi JY, Park YS, Jung HY, et al. Feasibility of endoscopic resection in superficial esophageal squamous carcinoma. Gastrointest Endosc 2011;73:881-889. 
12. Vazquez-Sequeiros E, Wiersema MJ, Clain JE, et al. Impact of lymph node staging on therapy of esophageal carcinoma. Gastroenterology 2003;125:1626-1635.

13. Vazquez-Sequeiros E, Levy MJ, Clain JE, et al. Routine vs. selective EUS-guided FNA approach for preoperative nodal staging of esophageal carcinoma. Gastrointest Endosc 2006;63:204-211.

14. Kelly S, Harris KM, Berry E, et al. A systematic review of the staging performance of endoscopic ultrasound in gastro-oesophageal carcinoma. Gut 2001;49:534-539.

15. Chen VK, Eloubeidi MA. Endoscopic ultrasound-guided fine needle aspiration is superior to lymph node echofeatures: a prospective evaluation of mediastinal and peri-intestinal lymphadenopathy. Am J Gastroenterol 2004;99:628-633.

16. Chen J, Xu R, Hunt GC, Krinsky ML, Savides TJ. Influence of the number of malignant regional lymph nodes detected by endoscopic ultrasonography on survival stratification in esophageal adenocarcinoma. Clin Gastroenterol Hepatol 2006;4:573-579.

17. Parmar KS, Zwischenberger JB, Reeves AL, Waxman I. Clinical impact of endoscopic ultrasound-guided fine needle aspiration of celiac axis lymph nodes (Mla disease) in esophageal cancer. Ann Thorac Surg 2002;73:916-920.

18. Eloubeidi MA, Wallace MB, Hoffman BJ, et al. Predictors of survival for esophageal cancer patients with and without celiac axis lymphadenopathy: impact of staging endosonography. Ann Thorac Surg 2001;72:212219.

19. Marsman WA, van Wissen M, Bergman JJ, et al. Outcome of patients with esophageal carcinoma and suspicious celiac lymph nodes as determined by endoscopic ultrasonography. Endoscopy 2004;36:961-965.

20. Reed CE, Mishra G, Sahai AV, Hoffman BJ, Hawes RH. Esophageal cancer staging: improved accuracy by endoscopic ultrasound of celiac lymph nodes. Ann Thorac Surg 1999;67:319-321.

21. Puli SR, Batapati Krishna Reddy J, Bechtold ML, Antillon MR, Ibdah JA. How good is endoscopic ultrasound for TNM staging of gastric cancers? A meta-analysis and systematic review. World J Gastroenterol 2008;14:4011-4019.

22. Mocellin S, Marchet A, Nitti D. EUS for the staging of gastric cancer: a meta-analysis. Gastrointest Endosc 2011;73:1122-1134.

23. Mouri R, Yoshida S, Tanaka S, Oka S, Yoshihara M, Chayama K. Usefulness of endoscopic ultrasonography in determining the depth of invasion and indication for endoscopic treatment of early gastric cancer. J Clin Gastroenterol 2009;43:318-322.

24. Holt B, Rösch T, Peter S. EUS in the evaluation of gastric tumors. In: Hawes RH, Fockens P, Varadarajulu S, eds. Endosonography. Philadelphia: Elsevier Saunders; 2015. p. 129-150.

25. Cardoso R, Coburn N, Seevaratnam R, et al. A systematic review and meta-analysis of the utility of EUS for preoperative staging for gastric cancer. Gastric Cancer 2012;15 Suppl 1:S19-S26.

26. Kwee RM, Kwee TC. Imaging in local staging of gastric cancer: a systematic review. J Clin Oncol 2007;25:2107-2116.

27. Kwee RM, Kwee TC. Imaging in assessing lymph node status in gastric cancer. Gastric Cancer 2009;12:6-22.

28. Wang Z, Chen JQ. Imaging in assessing hepatic and peritoneal metastases of gastric cancer: a systematic review. BMC Gastroenterol 2011;11:19.

29. Gleeson FC. EUS in rectal cancer. In: Hawes RH, Fockens P, Varadarajulu S, eds. Endosonography. Philadelphia: Elsevier Saunders; 2015. p. 260268.

30. Bipat S, Glas AS, Slors FJ, Zwinderman AH, Bossuyt PM, Stoker J. Rectal cancer: local staging and assessment of lymph node involvement with endoluminal US, CT, and MR imaging: a meta-analysis. Radiology 2004;232:773-783. 